\title{
Transplantation of Non-Hematopoietic Adult Bone Marrow Stem/ Progenitor Cells Isolated by the p75 Nerve Growth Factor Receptor into the Penis Rescues Erectile Function in a Rat Model of Cavernous Nerve Injury
}

\author{
Muammer Kendirci ${ }^{1,2,{ }^{*}}, \dagger$, Landon Trost ${ }^{2, \%}$, Benjamin Bakondi ${ }^{4}$, Mandolin J. Whitney ${ }^{3}$, \\ Wayne J.G. Hellstrom ${ }^{2}$, and Jeffrey L. Spees $3,4,{ }^{*}, \dagger$ \\ ${ }^{1}$ Department of Urology, Sisli Etfal Training and Research Hospital, Istanbul, Turkey \\ 2 Department of Urology, Tulane University, School of Medicine, New Orleans, LA \\ ${ }^{3}$ Center for Gene Therapy, Tulane University, School of Medicine, New Orleans, LA \\ ${ }^{4}$ Department of Medicine, Stem Cell Core, University of Vermont, Colchester, VT 05446
}

\section{Abstract}

Purpose-Radical prostatectomy to treat prostate cancer frequently results in erectile dysfunction and reduced quality-of-life. We investigated the effects of transplanting nonhematopoietic adult bone marrow stem/progenitor cells (multipotent stromal cells; MSCs) into the corpus cavernosum in a rat model of bilateral cavernous nerve crush injury (BCNCI).

\begin{abstract}
Materials and Methods-MSCs were isolated from the bone marrow of transgenic green fluorescent protein (GFP) rats by plastic adherence (rMSCs) or magnetic-activated cell sorting (MACS) using antibodies against the p75 low-affinity nerve growth factor receptor (p75LNGFR; p75-derived MSCs). BCNCI was induced in adult male Sprague-Dawley rats. Immediately after injury, rats were injected intracavernosally either with PBS (vehicle control, $n=8$ ), fibroblasts (cell control, $n=8$ ), rMSCs (cell treatment, $n=8$ ), or p75dMSCs (cell treatment, $n=8$ ). Other rats underwent a sham operation (PBS injection, $\mathrm{n}=8$ ). Four weeks after the procedures, erectile function was assessed by measurement of intracavernosal-to-mean arterial pressure ratios (ICP/ MAP) and total ICP values during stimulation of the cavernosal nerve.

Results-Intracavernous injection of p75dMSCs after BCNCI resulted in significantly higher mean ICP/MAP and total ICP values compared with all other groups except for sham $(\mathrm{p}<0.05)$. The animals injected with rMSCs had partial rescue of erectile function compared with animals that received p75dMSCs. Fibroblast (cell control) and PBS (vehicle control) injections did not improve erectile function. ELISA results suggested that basic fibroblast growth factor (bFGF) secreted by p75dMSCs protected the cavernosal nerve after BCNCI.
\end{abstract}

Conclusions-Transplantation of adult stem/progenitor cells may provide an effective treatment for ED following radical prostatectomy.

*Correspondence: Muammer Kendirci, M.D., Associate Professor of Urology, Şişli Etfal Egitim ve Araştirma Hastanesi, 2. Üroloji Kliniği 34377, Istanbul, Turkey, Phone: +90 (212) 2312209; Fax: +90 (212) 2339687; mkendirci@ superonline.com. Jeffrey L. Spees, Ph.D., Assistant Professor of Medicine, Director, Stem Cell Core University of Vermont, Colchester, VT 05546, Phone: (802) 6562388; Fax: (802) 656-8932; Jeffrey.Spees@uvm.edu.

${ }^{\%}$ Current Institution: Department of Urology, Mayo Clinic, Rochester, MN 55905

$\dagger$ These authors contributed equally to this work. 


\section{Keywords}

Erectile dysfunction; stem cell; progenitor cell; cavernous nerve injury

\section{INTRODUCTION}

Prostate cancer is the most commonly diagnosed and treated solid malignancy in American men. Currently there are 192,280 new cases diagnosed and 27,360 deaths attributed annually to prostate cancer. ${ }^{1}$ Approximately $40 \%$ of new cases each year undergo radical prostatectomy. ${ }^{2}$ Radical prostatectomy causes erectile dysfunction (ED) principally by damaging the neurovascular mechanism responsible for initiating erections. Oral phosphodiesterase type 5 (PDE-5) inhibitors and intracavernous vasoactive agents are commonly used to treat ED after radical prostatectomy, but they are unsuccessful in more than half of post-prostatectomy patients. ${ }^{3}$

Adult stem/progenitor cells have great potential to treat cavernous nerve injury. Adult bone marrow contains adult non-hematopoietic stem/progenitor cells that are commonly referred to as mesenchymal stem cells or multipotent stromal cells (MSCs). MSCs are easily isolated by bone marrow aspiration, expand extensively in culture, differentiate into multiple cell types, and can be used to repair injured tissues. They heal injured tissues, in part, by the secreting numerous growth factors/cytokines and hormones that are anti-apoptotic, antinecrotic, anti-inflammatory, pro-mitotic, and angiogenic. ${ }^{4,5}$

We investigated the effects of transplanting marrow-derived MSCs in a rat model of bilateral cavernous nerve crush injury (BCNCI). To develop a standardized cell therapy for $\mathrm{ED}$, we determined the effects of administering typical rat MSCs (rMSCs) isolated by adherence to cell culture plastic and also a subpopulation of MSCs isolated by magneticactivated cell sorting (MACS) against the p75 low-affinity nerve growth factor receptor (p75-derived MSCs, p75dMSCs). This receptor was previously shown to isolate multipotent MSCs from adult human bone marrow. ${ }^{6}$

\section{MATERIALS AND METHODS}

\section{Preparation of Cells}

MSCs were isolated from adult green fluorescent protein (GFP) transgenic rats under protocols approved by the Animal Care and Use Committee (IACUC) of Tulane University Health Sciences Center. Transgenic Sprague-Dawley rats that ubiquitously express enhanced GFP under control of the cytomegalovirus (CMV) enhancer and the chicken sactin promoter were provided by M. Okabe, Genome Information Research Center, Osaka University, Osaka, Japan. ${ }^{7}$

GFP-positive bone marrow cells were isolated from femurs and tibias of rats by capping the bones, placing the shafts into $1000 \mu \mathrm{l}$ pipet tips cut to fit into $1.5 \mathrm{ml}$ centrifuge tubes, and centrifuging the samples at $950 \times \mathrm{g}$ for 1 minute to pellet the marrow. Mononuclear bone marrow cells from pooled cell pellets were re-suspended into $25 \mathrm{ml}$ of Hanks Balanced Salt Solution (Gibco, Invitrogen, Carlsbad, CA), filtered (70 micron cell strainer, Thermo Fisher Scientific, Rochester, NY), and purified by density centrifugation at $1800 \times \mathrm{g}$ for 30 minutes without braking (Ficoll-Paque PLUS, GE Healthcare, Piscataway, NJ). To isolate GFPpositive rat MSCs (rMSCs), mononuclear cells were re-suspended in a complete culture medium (CCM) consisting of $\alpha$-MEM (Invitogen) with $20 \%$ fetal calf serum (lot selected for rapid growth of MSCs; Atlantic Biologicals, Norcross, GA), 100 units per ml penicillin, 100 $\mu \mathrm{g}$ per $\mathrm{ml}$ streptomycin, and $1 \mathrm{mM}$ L-glutamine (Invitrogen). Non-adherent cells were 
washed off after 24 hours with PBS and fresh CCM was added. As reported previously for rMSCs from normal Lewis rats, GFP rMSCs from transgenic Sprague-Dawley rats formed small colonies of rapidly growing spindle-shaped cells after about 1 week. ${ }^{8}$ For isolation of MSCs from GFP transgenic rats by the p75 low affinity nerve growth factor receptor (p75LNGFR), mononuclear cells were re-suspended in $0.4 \mathrm{ml}$ PBS containing $0.5 \%$ bovine serum albumin and $2 \mathrm{mM}$ EDTA (MACS buffer). After adding 5 micrograms $/ \mathrm{ml}$ mouse anti-rat p75LNGFR (MAB365, Chemicon, Millipore Corporation, Billerica, MA), the sample was incubated for 30 minutes at $4^{\circ} \mathrm{C}$. After 2 PBS washes, secondary antibody conjugated to magnetic (MACS) beads was added (100 microliters of goat anti-mouse IgG Microbeads; Miltenyi Biotech, Auburn, CA). The sample was incubated for 30 minutes at $4^{\circ} \mathrm{C}$, washed, and then applied to a magnetic column (LS Column; Miltenyi Biotech). The bound fraction was eluted with $5 \mathrm{ml}$ of MACS buffer and cells were concentrated by centrifugation at $600 \times \mathrm{g}$ for 10 minutes. After re-suspension, the entire isolate was cultured in $15 \mathrm{ml}$ of CCM in a T75 flask (Nunc, Thermo Fisher Scientific). MSC-like cells appeared as small colonies after 1 week. Adult skin fibroblasts were generated from skin biopsies taken from GFP rats that were used for bone marrow cell isolation. Cultured rMSCs, p75dMSCs, and dermal fibroblasts all had a mesodermal, fibroblastic morphology. Because GFP can be expressed at varying levels in different tissues and cell types, all cells to be used for transplantation were first grown to passage 3 and examined by epifluorescence microscopy (Zeiss Axiovert 200, Carl Zeiss MicroImaging, Inc., Thornwood, NY) to confirm the expression of GFP (Fig. 1). Passage 3-5 cells were used for all experiments.

\section{Induction of Bilateral Cavernous Nerve-Crush Injury}

Adult male Sprague-Dawley rats (300-350 g) underwent BCNCI. ${ }^{9}$ Briefly, after intraperitoneal injection of sodium pentobarbital $(50 \mathrm{mg} / \mathrm{kg})$ for anesthesia, animals were placed in a supine position on a heated surgical table. A lower midline abdominal incision was made after the abdomen was shaved and sterilized. The prostate gland was exposed and the cavernous nerves tracking posterolaterally were identified and isolated (see fig. 2). In sham-operated rats, there was no further surgical manipulation. In all other groups, BCNCI was performed with a hemostat clamp for 2 minutes. The abdomen was then closed in two layers in all rats.

\section{Transplantation of Cells into the Corpora Cavernosa}

Following BCNCI, the penis was exposed and $40 \mu \mathrm{l}$ of cell suspension in PBS $(500,000$ cells) or $40 \mu 1$ of PBS (vehicle) was injected into the corpus cavernosum with a 25-gauge needle (see fig. 2). Various groups of rats were injected with PBS (control, $n=8$ ), fibroblasts (cell control, $n=8$ ), rMSCs (cell treatment, $n=8$ ), or p75dMSCs (cell treatment, $n=8$ ). Shamoperated animals were injected with PBS $(\mathrm{n}=8)$. Immediately before injection, blood drainage via the dorsal vein was halted by circumferential compression of the penis at the base with an elastic band. The compression was released 1 minute after injection of cells or PBS.

\section{Measurement of Erectile Responses}

Four weeks after the intracavernous injections, the rats were anesthetized with an intraperitoneal injection of pentobarbital $(50 \mathrm{mg} / \mathrm{kg})$ and placed onto a thermally-regulated surgical table. The trachea was cannulated with PE-240 polyethylene tubing to maintain airway patency. A carotid artery was cannulated with PE-50 tubing to continuously measure systemic blood pressure (MAP) with a Statham transducer (Viggo Spectramed, Oxnard, CA) and a data acquisition system (Biopac Systems, Santa Barbara, CA). A 25G needle filled with heparin $(250 \mathrm{U} / \mathrm{mL})$ was attached to PE-50 tubing, inserted into the right crura, and connected to a pressure transducer to permit continuous measurement of ICP. The cavernous nerve $(\mathrm{CN})$ was identified posterolateral to the prostate on one side and an electrical 
stimulator with a stainless-steel bipolar hook was placed around the $\mathrm{CN}$, proximal to the injury site. The $\mathrm{CN}$ was stimulated with a square pulse stimulator (Grass Instruments, Quincy, MA). ${ }^{10}$ Measurements were made in a blinded fashion.

\section{Immunohistochemical Localization of Transplanted Cells}

Detailed immunohistochemistry procedures for transplanted GFP-positive cells were reported previously. ${ }^{11,12}$ To accurately detect engrafted cells in the corpus cavernosum we performed staining first with an unconjugated primary antibody against GFP and then with a secondary antibody that was conjugated to ALEXA 594 (red).

\section{ELISAs for secreted proteins}

GFP rMSCs, GFP p75dMSCs, and GFP fibroblasts were expanded to 90\% confluence in $150 \mathrm{~cm}^{2}$ dishes to generate conditioned medium (CdM). To prepare CdM, cells were washed 2 times with PBS, and then incubated in $20 \mathrm{ml}$ of fresh serum-free $\alpha$-MEM for 48 hours. The culture medium was collected, filtered $(0.22 \mu \mathrm{M}$ filter, Millipore, Billerica, MA), and stored at $-80^{\circ} \mathrm{C}$. Sandwich ELISAs for $\beta$-NGF, BDNF, VEGF, and IGF-1 were performed with commercially available kits (R and D Systems, Minneapolis, MN). The bFGF ELISA was performed directly using a biotinylated polyclonal primary antibody (ab12476-25, Abcam, Cambridge, MA).

\section{Statistical Analysis}

All data were expressed as the mean \pm standard error of the mean (SEM) and were analyzed using one-way ANOVA with repeated measures and Neumann-Kuels post hoc testing for multiple group comparisons with Statview (Abacus Concepts, Inc., Berkeley, CA). ELISA data were evaluated by Student's T Test (2-tailed). Values of $\mathrm{p}<0.05$ were considered statistically significant.

\section{RESULTS}

\section{Neurogenic-Mediated Erectile Responses Following Recovery from $\mathrm{BCNCI}$}

Figure $2 \mathrm{~A}$ and $\mathrm{B}$ show the magnitude of the mean ICP/MAP and total ICP values measured in response to CNS for 1 minute in all animal groups at 4 weeks after injury. BCNCI resulted in significantly lower mean ICP/MAP and total ICP values in PBS- and fibroblastinjected rats compared with those of sham-operated rats ( $p<0.05$, fig. 2$)$. The animals that received rMSC injections demonstrated significant improvement in erectile function compared with animals that received PBS or fibroblasts at 5.0 volts, but not at the other voltages tested (see 5.0 volts; $\mathrm{p}<0.05$, fig. 2). Rats that received p75dMSCs after BCNCI had significantly higher mean ICP/MAP and total ICP values compared with rats that received PBS, fibroblasts, or rMSCs ( $p<0.05$, fig. 2). Remarkably, in several instances, the animals that received p75dMSCs after BCNCI did not differ from sham-operated rats in their erectile function (fig. 2).

\section{Survival and Localization of GFP MSCs and GFP p75dMSCs In Vivo}

Four weeks after intracavernous injections, the survival of transplanted cells was determined by GFP immunohistochemistry (fig. 3). The surviving engrafted MSCs and p75dMSCs had a mesodermal (fibroblastic) rather than a neuronal morphology. We did not detect any GFPpositive cells in penile tissues of animals that received fibroblasts (data not shown). The rare long-term engraftment of rMSCs and p75MSCs and their morphology in vivo indicated that the beneficial effects of the stem/progenitor cell administrations were not likely the result of cell replacement though engraftment and differentiation (i.e. as neurons). 


\section{Phenotypic Characteristics of Cultured p75dMSCs}

To further characterize the p75dMSCs, we performed immunocytochemistry on cultured cells for proteins known to be expressed in fibroblasts, smooth muscle cells, and skeletal muscle cells (fig. 4 and data not shown).

\section{ELISAs for Neuro- and Vaso-Protective Secreted Proteins}

Bone marrow-derived stem/progenitor cells are well-known to secrete multiple growth factors and cytokines that protect cells, increase angiogenesis, and promote tissue repair after injury. ${ }^{4,5}$ Because of their potential to provide vaso- and neuroprotection after injury, we examined the secretion of basic Fibroblast Growth Factor (bFGF), beta Nerve Growth Factor ( $\beta$-NGF), Brain-Derived Neurotrophic Factor (BDNF), Vascular Endothelial Growth Factor (VEGF), and Insulin-like Growth Factor 1 (IGF-1) from fibroblasts, rMSCs, and p75dMSCs by ELISAs (fig. 5). The p75dMSCs secreted significantly more bFGF than did fibroblasts or rMSCs. The p75dMSCs secreted more $\beta$-NGF compared with rMSCs. In contrast, the rMSCs secreted more VEGF than did the p75dMSCs. The fibroblasts secreted more $\beta$-NGF and VEGF than did rMSCs or p75dMSCs. Both rat fibroblasts and rMSCs secreted detectable levels of IGF-1, but the p75dMSCs did not (fig. 5).

\section{DISCUSSION}

ED following radical prostatectomy causes significant morbidity that affects a large percentage of men. Although several hypotheses exist, one possible mechanism underlying post-prostatectomy ED involves an initial insult to the cavernosal neurovascular bundle that leads to hypoxia, apoptosis, and subsequent corporal fibrosis. ${ }^{13,14}$ Evidence for the role of neurovascular damage in post-prostatectomy ED is provided by the resultant decreases in ED following adoption of nerve-sparing techniques in radical prostatectomy. ${ }^{15}$

Compared with current treatment modalities, stem cells may provide a unique therapy for post-prostatectomy ED. Instead of focusing on improving erections at the level of the penis, currently the target of many penile rehabilitation pharmacotherapies, the administration of stem cells provides treatment at the level of the cavernosal nerve. Infusion of MSCs often down-regulates or alters immune responses; this property justifies their allogeneic use in some cases. Alternatively, as much of their effects may result from transient paracrine activity, immune matching may not be necessary to achieve substantial treatment benefits with MSCs. Bivalacqua and colleagues examined the effect of MSCs alone or endothelial nitric oxide synthase (eNOS)-gene modified MSCs on erectile function in aged rats. ${ }^{16}$ Their results demonstrated improved erectile function after 7 days in rats treated with eNOSmodified MSCs, and at day 21 in rats treated with unmodified MSCs.

In our results, at 1 month after BCNCI, rats injected with p75dMSCs showed significant increases in mean ICP/MAP and total ICP values compared with rats that received PBS, fibroblasts or rMSCs in response to the cavernosal nerve stimulation at the proximal site of the injured nerve for 1 minute at all electrical stimulations tested, displaying complete rescue of erectile function. Interestingly, rats treated with rMSCs also demonstrated significant improvement in mean ICP/MAP and total ICP values over PBS- and fibroblastinjected animals as evidenced by 5 -volt stimulation (optimal stimulation dose), but failed to demonstrate similar statistical differences at 2.5 (sub-physiological) and 7.5 volts (supraphysiological). Similar to the case with p75dMSCs, improvement in the rMSC group may have been due to paracrine action of the transplanted cells.

Since the BCNCI model is essentially a neurogenic-mediated model of ED, it can be inferred that injection of rMSCs and p75dMSCs most likely resulted in improved nerve function through either the inhibition of nerve damage (apoptosis or necrosis occurring after 
nerve crush) or through enhanced nerve regeneration. The small numbers of transplanted cells present at 4 weeks after injection supported a paracrine mechanism. Accordingly, we examined the secretion of bFGF, $\beta$-NGF, BDNF, VEGF, and IGF-1, growth factors that had previously been shown to reduce ED in diabetic rats, ${ }^{17,18}$ aged rats,,${ }^{19,20}$ and in rats with cavernous nerve injury. ${ }^{21-23}$ Of all the secreted proteins we measured by ELISA, bFGF was the most highly secreted growth factor by p75dMSCs. This growth factor is a promising candidate to investigate further as it is well known to be neuroprotective for both the central and peripheral nervous systems. ${ }^{24,25}$ In addition, bFGF has been identified as one of the principal neurotrophic factors produced within the penis. ${ }^{26}$ In future studies, we will evaluate the relative importance of bFGF secreted by p75dMSCs with lentiviral shRNA knockdowns and treatment of the BCNCI model with depleted and control p75dMSCs.

The dramatic improvement in erectile function following treatment with the p75dMSCs from adult bone marrow suggests that they may provide an effective treatment to reduce ED post-prostatectomy and perhaps also for other injuries that result in ED. Although the cell treatments in our study were allogeneic, because radical prostatectomy is a scheduled surgery p75dMSCs could be easily isolated from a bone marrow aspirate, expanded for 2 weeks prior to surgery, and used also in an autologous manner.

\section{CONCLUSIONS}

Non-hematopoietic p75dMSCs from bone marrow may provide a treatment to reduce ED after cavernous nerve injury.

\section{Acknowledgments}

This manuscript was supported in part by grants from SMSNA/Bayer GSK (MK) and the National Institutes of Health: NHLBI HL077570 (JLS), NHLBI HL085210 (JLS), and P20 RR016435 NIH/NCRR (Parsons R, COBRE PI, JLS, PI project 3).

\section{References}

1. Jemal A, Siegel R, Ward E, et al. Cancer statistics, 2009. CA Cancer J Clin 2009;59:225-249. [PubMed: 19474385]

2. Kendirci M, Hellstrom WJG. Restoring erectile function: Proactive options after radical prostatectomy. Contemp Urol 2005;17:36.

3. Kendirci M, Hellstrom WJ. Current concepts in the management of erectile dysfunction in men with prostate cancer. Clin Prostate Cancer 2004;3:87-92. Review. [PubMed: 15479491]

4. Phinney DG, Prockop DJ. Concise review: mesenchymal stem/multipotent stromal cells: the state of transdifferentiation and modes of tissue repair--current views. Stem Cells 2007;25:2896-2902. Review. [PubMed: 17901396]

5. Gnecchi M, Zhang Z, Ni A, Dzau VJ. Paracrine mechanisms in adult stem cell signaling and therapy. Circ Res 2008;103:1204-1219. Review. [PubMed: 19028920]

6. Quirici N, Soligo D, Bossolasco P, et al. Isolation of bone marrow mesenchymal stem cells by antinerve growth factor receptor antibodies. Exp Hematol 2002;30:783-791. [PubMed: 12135677]

7. Ito T, Suzuki A, Imai E, et al. Bone marrow is a reservoir of repopulating mesangial cells during glomerular remodeling. J Am Soc Nephrol 2001;12:2625-2635. [PubMed: 11729231]

8. Javazon EH, Colter DC, Schwarz EJ, et al. Rat marrow stromal cells are more sensitive to plating density and expand more rapidly from single-cell-derived colonies than human marrow stromal cells. Stem Cells 2001;19:219-225. [PubMed: 11359947]

9. Kendirci M, Zsengeller Z, Bivalacqua TJ, et al. Poly(Adenosine diphosphate-ribose) polymerase inhibition preserves erectile function in rats after cavernous nerve injury. J Urol 2005;174:20542059. [PubMed: 16217393] 
10. Bivalacqua TJ, Usta MF, Champion HC, et al. Gene transfer of endothelial nitric oxide synthase partially restores nitric oxide synthesis and erectile function in streptozotocin diabetic rats. J Urol 2003;169:1911-1917. [PubMed: 12686872]

11. Spees JL, Pociask DA, Sullivan DE, et al. Engraftment of bone marrow progenitor cells in a rat model of asbestos-induced pulmonary fibrosis. Am J Respir Crit Care Med 2007;176:385-394. [PubMed: 17496228]

12. Spees JL, Whitney MJ, Sullivan DE, et al. Bone marrow progenitor cells contribute to repair and remodeling of the lung and heart in a rat model of progressive pulmonary hypertension. FASEB J 2008;22:1226-1236. [PubMed: 18032636]

13. Mulhall JP, Graydon RJ. The hemodynamics of erectile dysfunction following nerve-sparing radical retropubic prostatectomy. Int J Impot Res 1996;8:91-94. [PubMed: 8858398]

14. Mulhall JP, Slovick R, Hotaling J, et al. Erectile dysfunction after radical prostatectomy: hemodynamic profiles and their correlation with the recovery of erectile function. $\mathrm{J}$ Urol 2002;167:1371-1375. [PubMed: 11832735]

15. Catalona WJ, Basler JW. Return of erections and urinary continence following nerve sparing radical retropubic prostatectomy. J Urol 1993;150:905-907. [PubMed: 8345607]

16. Bivalacqua TJ, Deng W, Kendirci M, et al. Mesenchymal stem cells alone or ex vivo gene modified with endothelial nitric oxide synthase reverse age-associated erectile dysfunction. Am J Physiol Heart Circ Physiol 2007;292:H1278-1290. [PubMed: 17071732]

17. Suetomi T, Hisasue S, Sato Y, Tabata Y, Akaza H, Tsukamoto T. Effect of basic fibroblast growth factor incorporating gelatin microspheres on erectile function in the diabetic rat. J Urol 2005;173:1423-1428. [PubMed: 15758817]

18. Yamanaka M, Shirai M, Shiina H, et al. Vascular endothelial growth factor restores erectile function through inhibition of apoptosis in diabetic rat penile crura. J Urol 2005;173:318-323. [PubMed: 15592104]

19. Park K, Ahn KY, Kim MK, Lee SE, Kang TW, Ryu SB. Intracavernosal injection of vascular endothelial growth factor improves erectile function in aged rats. Eur Urol 2004;46:403-407. [PubMed: 15306114]

20. Pu XY, Wang XH, Gao WC, et al. Insulin-like growth factor-1 restores erectile function in aged rats. modulation the integrity of smooth muscle and nitric oxide-cyclic guanosine monophosphate signaling activity. J Sex Med 2008;5:1345-1354. [PubMed: 18355170]

21. Burgers JK, Nelson RJ, Quinlan DM, Walsh PC. Nerve growth factor, nerve grafts and amniotic membrane grafts restore erectile function in rats. J Urol 1991;146:463-468. [PubMed: 1856953]

22. Hsieh PS, Bochinski DJ, Lin GT, Nunes L, Lin CS, Lue TF. The effect of vascular endothelial growth factor and brain-derived neurotrophic factor on cavernosal nerve regeneration in a nervecrush rat model. BJU Int 2003;92:470-475. [PubMed: 12930443]

23. Chen KC, Minor TX, Rahman NU, Ho HC, Nunes L, Lue TF. The additive erectile recovery effect of brain-derived neurotrophic factor combined with vascular endothelial growth factor in a rat model of neurogenic impotence. BJU Int 2005;95:1077-1080. [PubMed: 15839936]

24. Alzheimer C, Werner S. Fibroblast growth factors and neuroprotection. Adv Exp Med Biol 2002;513:335-51. Review. [PubMed: 12575827]

25. Grothe C, Haastert K, Jungnickel J. Physiological function and putative therapeutic impact of the FGF-2 system in peripheral nerve regeneration--lessons from in vivo studies in mice and rats. Brain Res Rev 2006;51:293-299. Review. [PubMed: 16430964]

26. Te AE, Santarosa RP, Koo HP, et al. Neurotrophic factors in the rat penis. J Urol 1994;152:21672172. [PubMed: 7966709] 

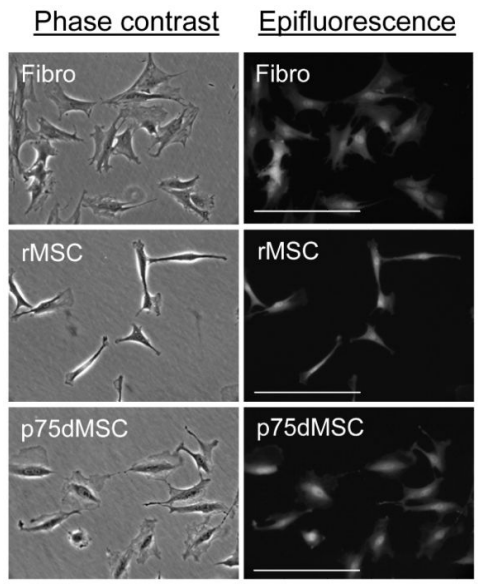

Figure 1.

Morphology of cultured GFP rat fibroblasts (Fibro), GFP rat MSCs (rMSC), and GFP rat p75-derived MSCs (p75dMSC). The left column is phase contrast and the right column is epifluorescence microscopy for GFP (FITC channel). Scale bars= 100 micrometers. 


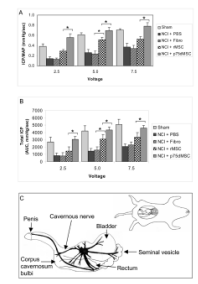

Figure 2.

Determination of intracavernous-to-mean arterial pressure ratio (ICP/MAP) and total ICP values to assess erectile function at 4 weeks after injury and treatment. A, Magnitude of the mean ICP/MAP. B, Mean total ICP. Cavernous nerve stimulation (CNS) at a frequency of $15 \mathrm{~Hz}$. and pulse width of 30 milliseconds was performed in each rat. CNS at 2.5, 5.0 and $7.5 \mathrm{~V}$ was performed in the current protocol to achieve significant and consistent erectile responses. The duration of stimulation was 1 minute with a rest period of 3 to 5 minutes between subsequent CNS episodes. The total erectile response or total ICP was determined by the area under the curve (AUC) in $\mathrm{mmHg} / \mathrm{second}$ from the beginning of CNS until the ICP returned to baseline or pre-stimulation pressure. The mean ICP-to-blood pressure ratio (ICP/MAP) at the peak erectile response was determined to control for variations in systemic blood pressure. C, Diagram of cavernous nerve anatomy and area of NCI. * p $<0.05$. Note: in all cases, PBS and fibro are significantly less than sham $(p<0.05)$ and p75dMSC is significantly greater than PBS and fibro ( $p<0.05)$. NCI: Bilateral cavernous nerve-crush injury. $\mathrm{N}=8$ animals per group. 


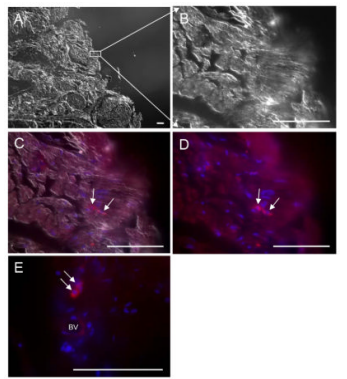

Figure 3.

The survival of MSCs in the penile sections using differential interference contrast (DIC) to define tissue morphology and epifluorescence to localize GFP. A, DIC image from edge of penis section. B, DIC image from edge of penis section. C, DIC image merged with staining by anti-GFP antibody (ALEXA 594, red, TRITC channel) and DAPI to visualize cell nuclei (blue). D, Epifluoresence alone from image in (C) showing signals for GFP and DAPI. E, Epifluoresence image from a different p75dMSC-injected rat in which the engrafted cells were located in close proximity to a blood vessel. BV: Blood vessel. Scale bars $=100$ micrometers. 

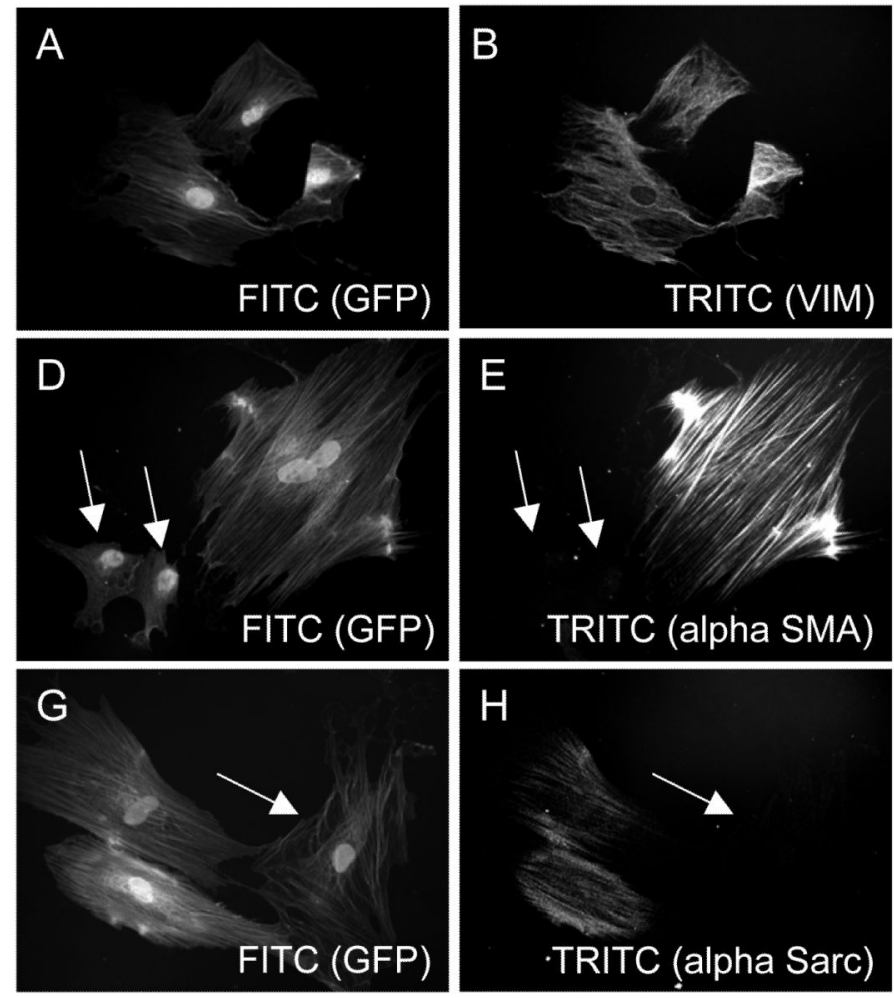
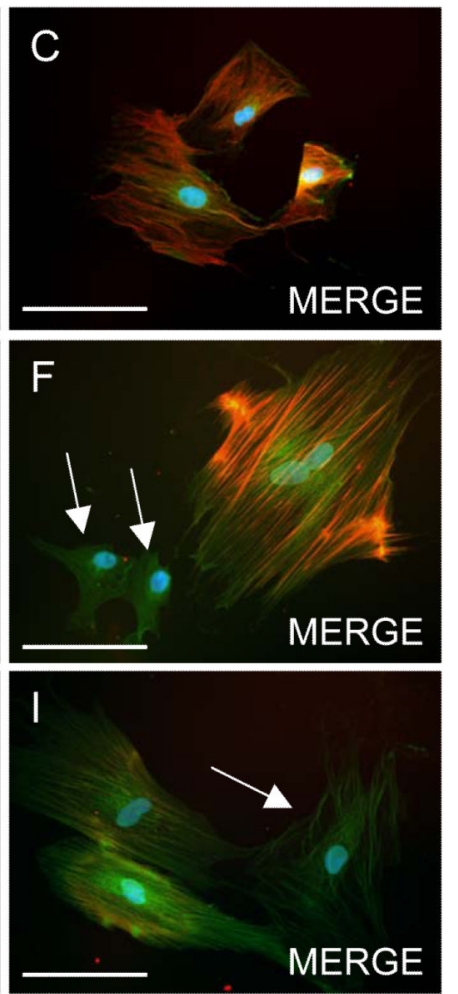

Figure 4.

Phenotypic characteristics of cultured p75dMSCs. A-C, By immunohistochemistry, similar to rMSCs and fibroblasts, all of the p75dMSCs expressed the intermediate filament protein vimentin (ALEXA 594, red, TRITC channel). D-F, Also similar to rMSCs and fibroblasts, some of the p75dMSCs expressed the myofibroblast and smooth muscle marker, alpha smooth muscle actin (ALEXA 594, red, TRITC channel). G-I, In contrast to rMSCs and fibroblasts, a portion of the p75dMSCs expressed alpha sarcomeric actin, a marker of cardiac or skeletal muscle cells. Arrows: GFP-positive cells (FITC channel) that are negative for the immunostains (TRITC channel). Scale bars= 100 micrometers. 


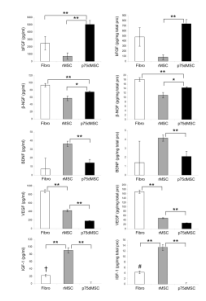

Figure 5.

ELISA data for secreted bFGF, $\beta$-NGF, BDNF, VEGF, and IGF-1 from fibroblasts, rMSCs, and p75dMSCs. All growth factor levels are shown as expressed per volume $(\mathrm{pg} / \mathrm{ml})$ on the left and per total protein (pg/mg total protein) on the right. * p <0.05, ** p <0.01, $\dagger \mathrm{p}<0.05$ vs. p75dMSC, \# p <0.01 vs. p75dMSC. $\mathrm{N}=3$ samples per cell type. 